\title{
Propagation of a solitary wave along a magnetic field in a cold collision-free plasma
}

\author{
By P. G. SAFFMAN \\ King's College, London
}

(Received 10 February 1961)

It is shown that solitary hydromagnetic waves can propagate parallel to a uniform magnetic field in a cold collision-free plasma. These waves are exact solutions of the non-linear equations of motion except for the quasi-neutral approximation. The velocity of propagation lies in a range of values somewhat larger than the Alfvén velocity, and is of the order of 25 times the Alfvén velocity for hydrogen, the precise value depending upon the strength of the wave. Simple expressions exist for the velocities of the ions and electrons and the magnetic field inside the wave. The lines of force are spirals about the direction of propagation. The waves are symmetrical about their middle. The order of magnitude of their width is the geometric mean of the gyro-radii of the ions and electrons when moving with the Alfvén velocity. The maximum value of the magnetic field can be somewhat larger than the value away from the wave.

\section{Introduction}

The propagation of finite-amplitude waves across a magnetic field in a cold collision-free plasma has been investigated by Adlam \& Allen (1958) and Davis, Lüst \& Schlüter (1958). One-dimensional solutions were obtained which describe the motion of a pulse or solitary wave in a direction perpendicular to a magnetic field, all quantities being uniform in planes perpendicular to the direction of propagation. The motion is steady relative to an observer moving with the wave. Davis et al. also obtained solutions describing periodic waves comprising an infinite wave train. The particular interest of the solitary wave solutions lies in the belief that they may provide zero-order approximations to collision-free shocks which propagate transversely to a magnetic field.

The similar problem of the propagation of finite-amplitude one-dimensional waves parallel to a magnetic field (as opposed to across) has been studied by Ferraro (1956) and Montgomery (1959). Their solutions, however, all described periodic waves of infinite extent. In the present note, it will be shown that solutions of the equations exist which represent solitary waves, which are perhaps of more intrinsic interest as being possibly relevant to collision-free shocks which propagate along a magnetic field.

In all this work, it is assumed that the plasma is approximately electrically neutral. That is, the number density of ions and electron are assumed equal, except in the Maxwell equation for the electric displacement. This assumption is valid provided all velocities are non-relativistic and any frequency is small 
compared with the plasma frequency. In fact, it is easy to show that the approximation is exact if the ion and electron masses are equal; and in the general case the waves are modified very slightly by the deviations from strict neutrality which actually occur.

\section{The equations of motion}

We consider now the existence of solitary waves in a cold collision-free plasma which propagate steadily with velocity $U$ along a magnetic field. We suppose that at infinity ahead of the wave conditions are uniform, the magnetic field is of strength $H_{0}$, and the number density of ions and electrons is $N$. Then, in a frame of reference moving with the wave, the motion is steady, all quantities are functions of $x$, the co-ordinate parallel to the magnetic field at infinity, and the velocity of ions and electrons at infinity is $U$ parallel to the $x$-axis.

We employ Gaussian units. Then all quantities can be made non-dimensional by the use of a characteristic velocity $U$, a characteristic number density $N$, a characteristic magnetic intensity $H_{0}$, and a characteristic length

$$
l=\frac{a c \sqrt{\left(m_{i} m_{e}\right)}}{e \bar{H}_{0}},
$$

where $e$ is the electronic charge, $m_{i}$ and $m_{e}$ are the masses of ions and electrons respectively, $c$ is the velocity of light, and

$$
a=\sqrt{\left[\frac{H_{0}^{2}}{4 \pi N\left(m_{i}+m_{e}\right)}\right]}
$$

is the Alfven velocity in the undisturbed plasma. The length $l$ is the geometric mean of the gyro-radii of the ions and electrons when moving with the Alfvén velocity.

Let $n$ denote the dimensionless number density of the ions and electrons (assumed equal by the quasi-neutral approximation). Then the equations of continuity show that the $x$-components of the velocity of the ions and electrons are equal; and denoting this (dimensionless) velocity by $u$, we have from the equations of continuity

$$
n u=1 .
$$

The conditions at infinity require that $n \rightarrow 1$ and $u \rightarrow 1$ as $x \rightarrow-\infty$.

It follows from Maxwell's equations with the particular geometry of this problem that only the $x$-component of the electric field is non-zero and that the $x$-component of the magnetic field is constant. Then the components of the (dimensionless) magnetic field can be written $\left(1, h_{2}, h_{3}\right)$, and those of the electric field $(E, 0,0)$, the actual electric field being $E U H_{0} / c$. We denote the (dimensionless) velocity of the ions by $\left(u, v_{i}, w_{i}\right)$ and that of the electrons by $\left(u, v_{e}, w_{e}\right)$. Then the equations of motion and Maxwell's equations (Montgomery 1959) roduce to

$$
\begin{array}{ll}
\frac{M}{\gamma} u \frac{d u}{d x}=E-w_{i} h_{2}+v_{i} h_{3}, & M \gamma u \frac{d u}{d x}=-E+w_{e} h_{2}-v_{e} h_{3} ; \\
\frac{M}{\gamma} u \frac{d v_{i}}{d x}=-u h_{3}+w_{i}, & M \gamma u \frac{d v_{e}}{d x}=u h_{3}-w_{e}
\end{array}
$$




$$
\begin{aligned}
\frac{M}{\gamma} u \frac{d w_{i}}{d x} & =u h_{2}-v_{i}, & M \gamma u \frac{d w_{e}}{d x} & =-u h_{2}+v_{e} ; \\
\frac{d h_{2}}{d x} & =\frac{M n}{\gamma+\gamma^{-1}}\left(w_{i}-w_{e}\right), & \frac{d h_{3}}{d x} & =-\frac{M n}{\gamma+\gamma^{-1}}\left(v_{i}-v_{e}\right) .
\end{aligned}
$$

Here, $M=U / a$ can be called the Alfvén Mach number, and $\gamma^{2}=m_{e} / m_{i}$ is the ratio of the particle masses. Altogether, we have nine equations for nine unknowns, and we are interested in solutions for which all variables except $u$ and $n$ tend to zero as $x \rightarrow-\infty$.

These equations are valid provided $u$ never changes sign, that is, provided the particle trajectories are not looped.

\section{Reduction of the equations of motion}

Simple integrals of these equations can easily be obtained. From (2.6) and (2.7), and the conditions at infinity, it follows that

$$
\begin{aligned}
& h_{3}=\frac{M^{2}}{\gamma+\gamma^{-i}}\left(\frac{w_{i}}{\gamma}+\gamma w_{e}\right), \\
& h_{2}=\frac{M^{2}}{\gamma+\gamma^{-1}}\left(\frac{v_{i}}{\gamma}+\gamma v_{e}\right) .
\end{aligned}
$$

Eliminating $E$ from (2.4) and substituting for $h_{2}$ and $h_{3}$, we obtain

$$
\left(\gamma+\gamma^{-1}\right) u \frac{d u}{d x}=M\left(v_{i} w_{e}-v_{e} w_{i}\right)
$$

Now, from $(2.5 a)$ and $(2.6 a)$,

$$
\begin{aligned}
& \frac{M}{\gamma}\left(u_{i} \frac{d v_{i}}{d x}+w_{i} \frac{d w_{i}}{d x}\right)=w_{i} h_{2}-v_{i} h_{3} \\
&=\frac{M^{2} \gamma}{\gamma+\gamma^{-1}}\left(w_{i} v_{e}-w_{e} v_{i}\right)=-M \gamma u \frac{d u}{d x} . \\
& v_{i}^{2}+w_{i}^{2}=\gamma^{2}\left(1-u^{2}\right), \\
& v_{e}^{2}+w_{e}^{2}=\gamma^{-2}\left(1-u^{2}\right) .
\end{aligned}
$$

Hence

and similarly

These equations show that in a real solution $u$ must be less than one.

Equation (3.3) ean also be written

$$
M^{2} \frac{d u}{d x}+h_{2} \frac{d h_{2}}{d x}+h_{3} \frac{d h_{3}}{d x}=0
$$

Hence

$$
M^{2} u+\frac{1}{2} h_{2}^{2}+\frac{1}{2} h_{3}^{2}=M^{2},
$$

by virtue of the conditions at infinity. This last integral may be interpreted physically as the conservation of momentum flux.

We can now obtain an equation for $u$. Substitute (3.1) and (3.2) for $h_{2}$ and $h_{3}$ in (3.6), and use (3.3), (3.4), (3.5) and the identity

$$
\begin{array}{cc}
\left(v_{i} w_{e}-v_{e} w_{i}\right)^{2}+\left(v_{i} v_{e}+w_{i} w_{e}\right)^{2} \equiv\left(v_{i}^{2}+w_{i}^{2}\right)\left(v_{e}^{2}+w_{e}^{2}\right), \\
\text { to obtain } \quad\left(u \frac{d u}{d x}\right)^{2}=(1-u)^{2}\left\{2(1+u)-\frac{\left(\gamma+\gamma^{-1}\right)^{2}}{M^{2}}\right\} .
\end{array}
$$


When this equation is solved for $u$, the magnitudes of the transverse velocities of the ions and electrons and the transverse magnetic field follow immediately from (3.4), (3.5) and (3.6).

The orientation of the transverse components can be found without difficulty. If we write

$$
\tan \theta=h_{3} / h_{2}, \quad \tan \theta_{i}=w_{i} / v_{i}, \quad \tan \theta_{e}=w_{e} / v_{e},
$$

then combination of equations (2.7) with the equations of this section gives

$$
u \frac{d \theta}{d x}=\frac{1}{2 M}\left(\gamma^{-1}-\gamma\right)
$$

and moreover $\quad \theta=\frac{1}{2}\left(\theta_{i}+\theta_{e}\right), \quad \cos \frac{1}{2}\left(\theta_{i}-\theta_{e}\right)=\frac{\gamma+\gamma^{-1}}{M[2(1+u)]^{\frac{1}{2}}}$.

\section{Structure of the solitary wave}

Equation (3.7) is symmetrical in $x$, so the solution can only be a solitary wave, with conditions the same ahead of and behind the wave, and not a shock. The condition that it represents a real wave is that the expression in curly brackets in (3.7) is positive for $u=1$ and vanishes for some value of $u$ between 0 and 1 . This condition requires

$$
\frac{\gamma+\gamma^{-1}}{\sqrt{2}}>M>\frac{\gamma+\gamma^{-1}}{2}
$$

or, in terms of dimensional quantities,

$$
\frac{H_{0}^{2}\left(m_{i}+m_{e}\right)}{8 \pi N m_{i} m_{e}}>U^{2}>\frac{H_{0}^{2}\left(m_{i}+m_{e}\right)}{16 \pi N m_{i} m_{e}} .
$$

where $U$ is the velocity of propagation of the waves referred to a frame in which the plasma is at rest at infinity. The upper limit on $U$ expresses the condition that the trajectories are not looped.

Simple expressions for the wave structure can be obtained by introducing a new independent variable $t$ by the relation

$$
d x / d t=u \text {. }
$$

Here $t$ is the time for a particle to go a distance $x$. Then (3.7) can be integrated immediately to give

and

$$
\begin{aligned}
u & =1-2 \lambda^{2} \operatorname{Bech}^{2} \lambda t \\
x & =t-2 \lambda \tanh \lambda t \\
\lambda^{2} & =1-\frac{\left(\gamma+\gamma^{-1}\right)^{2}}{4 M^{2}}
\end{aligned}
$$

where

The values of all the other variables follow immediately. In particular,

and

$$
\begin{gathered}
h_{\mathrm{2}}^{2}+h_{\mathrm{g}}^{2}=4 M^{2} \lambda^{2} \operatorname{sech}^{2} \lambda t, \\
\theta=\frac{1}{2 \bar{M}}\left(\gamma^{-1}-\gamma\right) t .
\end{gathered}
$$

Thus the component of the magnetic field in the plane of the wave rotates at a constant rate following a particle. 
The dimensional thickness of the waves is of the order of the characteristic length $l$, as is the case for solitary waves moving across a magnetic field. It is to be noted that since the value of $\gamma \simeq \frac{1}{40}$, the waves move much faster than linearized Alfvén waves in the plasma. This is very different from the case for transverse waves, whose velocity of propagation lies between $a$ and $2 a$.

These solitary waves are the only non-trivial solutions of the equations of motion for the case in which the plasma and magnetic field are uniform ahead of the wave and the magnetic field is parallel to the direction of propagation. They do not in themselves provide a collision-free shock, since conditions behind the wave are identical with those ahead. However, it is intended to investigate in a subsequent paper the case in which the magnetic field is inclined at an angle to the direction of propagation. It is hoped to show that in this case there may be more than one type of solution, and moreover that there are solutions with shock-like properties for which conditions at downstream infinity differ from those at upstream infinity.

I am grateful to Dr J. A. Shercliff for a suggestion which led to the present work.

\section{REFERENCES}

Adlam, J. H. \& Aluer, J. E. 1958 Phil. Mag. 3, 448.

DAvis, L., LÜST, R. \& SchLÜTER, A. 1958 Z. Naturforsch. $13 a, 916$.

Ferraro, V. C. A. 1955 Proc. Roy. Soc. A, 233, 310.

Montgomery, D. 1959 Phys. Fluids, 2, 585. 